\title{
MAJORANA NEUTRINO SEARCHES AT THE LHCb EXPERIMENT*
}

\author{
Anna Ossowska \\ on behalf of the LHCb Collaboration \\ H. Niewodniczański Institute of Nuclear Physics, Polish Academy of Sciences \\ Radzikowskiego 152, 31-342 Kraków, Poland \\ (Received April 23, 2018) \\ These proceedings present a set of searches for Majorana neutrinos, \\ performed using decays of $B$ and $D$ mesons to final states with a pair of \\ the same-sign leptons, using data collected by the LHCb experiment. No \\ signal of Majorana neutrinos is observed for any of these decays and upper \\ limits on the branching fractions are set.
}

DOI:10.5506/APhysPolB.49.1301

\section{Introduction}

The Standard Model (SM) of particle physics is one of the most successful theories in physics which describes the fundamental interactions between elementary particles. Half of the leptons in the SM are neutrinos. The observation of neutrino oscillations is evidence for lepton flavour violation (forbidden in the SM) and implies that neutrinos have non-zero mass, but their nature is still unknown. Neutrinos can be their own anti-particles, in that case they are called "Majorana" neutrinos or Dirac particles, with particles and antiparticles being different objects [1].

Lepton number/flavour violating decays of $B$ and $D$ mesons are forbidden in the Standard Model (SM), but could occur through the production of a Majorana neutrino. The observation of such decay would be a clear sign of new physics.

\section{The $\mathrm{LHCb}$ detector}

The $\mathrm{LHCb}$ detector $[2,3]$ shown in Fig. 1 is a single-arm forward spectrometer covering the pseudorapidity range $2<\eta<5$, designed to study

* Presented at the Cracow Epiphany Conference on Advances in Heavy Flavour Physics, Kraków, Poland, January 9-12, 2018. 
decays of particles containing $b$ and $c$ quarks. The detector includes a high precision tracking system consisting of a silicon-strip vertex detector (VELO) surrounding the $p p$ interaction region. The tracking system allows to reconstruct the trajectories of charged particles and to measure their momenta. The spectrometer magnet, required for the momentum measurement of charged particles, is a warm dipole magnet providing an integrated field of about $4 \mathrm{Tm}$, which bends charged particles in the horizontal plane. Charged hadrons are identified using two ring-imaging Cherenkov detectors, designed to identify particles associated to low-momentum and highmomentum tracks. The electromagnetic and hadronic calorimeters provide measurements of the energy of electrons, photons and hadrons. Muons are identified by a system of alternating layers of iron and multiwire proportional chambers. The on-line event selection is performed by a trigger system that includes a hardware stage, based on information from the calorimeter and muon systems, followed by a software stage.

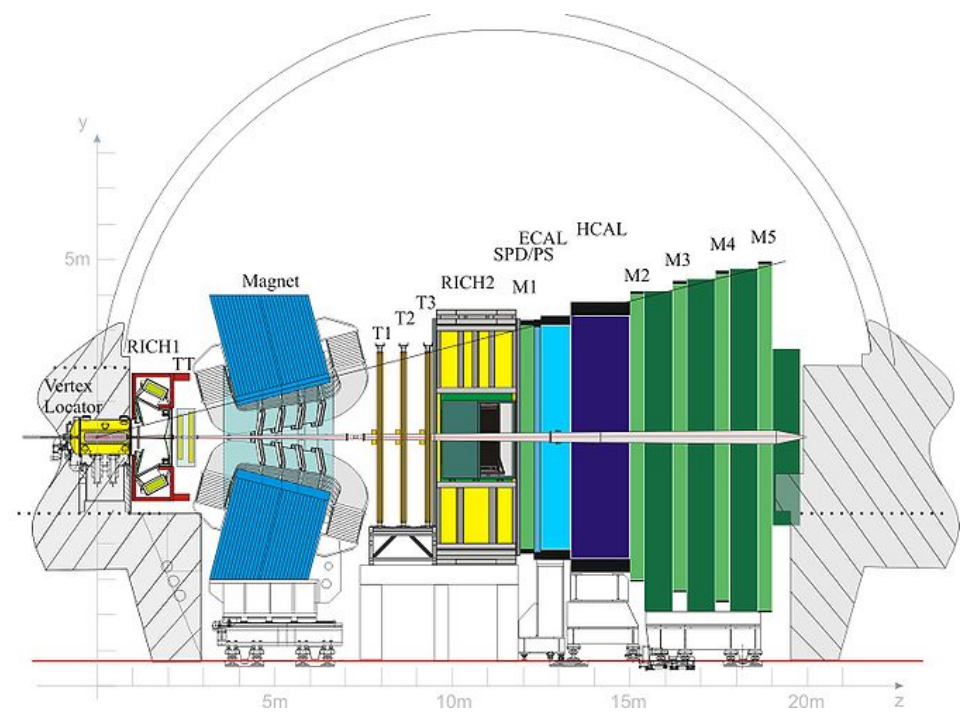

Fig. 1. The model of the $\mathrm{LHCb}$ detector [3].

\section{Chosen $B$ - and $D$-meson decays}

3.1. The search for $B^{-} \rightarrow \pi^{+} \mu^{-} \mu^{-}$decay

The decay $B^{-} \rightarrow \pi^{+} \mu^{-} \mu^{-}$(Fig. 2) allows to probe neutrino masses of up to $5 \mathrm{GeV} / c^{2}$. The total lepton number is violated, and so the decay is forbidden in the SM. The data used for this analysis corresponds to an integrated luminosity of $3 \mathrm{fb}^{-1}$ and was collected at a center-of-mass energy of 7 (8) $\mathrm{TeV}$ in 2011 (2012) [4]. 


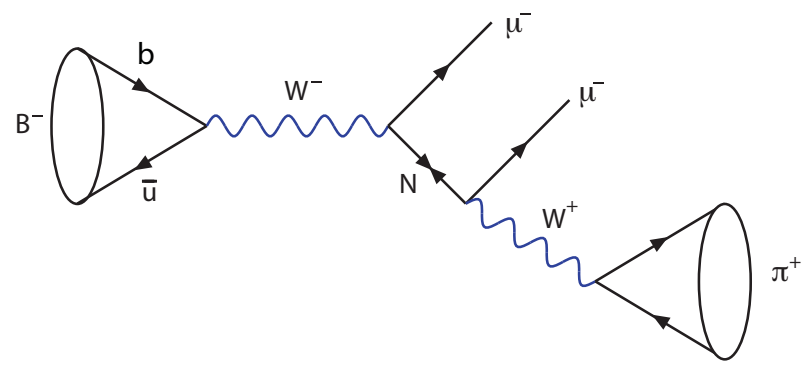

Fig. 2. The Feynman diagram of $B^{-} \rightarrow \pi^{+} \mu^{-} \mu^{-}$decay [4].

The $B^{-} \rightarrow J / \psi K^{-}$decay is used as normalisation channel. The hypothetical neutrino lifetime is scanned in the range of $0-1000 \mathrm{ps}$ split into two ranges. For lifetime $\tau>1 \mathrm{ps}$, the $\pi^{+} \mu^{-}$decay products can appear as significantly detached from the $B^{-}$decay vertex. Therefore, the analysis was performed in two strategies: one for short lifetime and another for lifetime up to $1000 \mathrm{ps}[4,6]$. This analysis used tracks that begin in the VELO for both of the strategies.

Figure 3 shows the mass spectra of the selected candidates. Backgrounds in the $\pi^{+} \mu^{-} \mu^{-}$final state come from $B$ decay to charmonium and combinatoric sources. No statistical excess of signal events is observed at any mass hypothesis, and upper limits on the branching fraction are set by $\mathrm{CL}_{s}$ method. For neutrino lifetimes shorter than $1 \mathrm{ps}$, an upper limit on $\mathcal{B}\left(B^{-} \rightarrow \pi^{+} \mu^{-} \mu^{-}\right)$is set

$$
\mathcal{B}\left(B^{-} \rightarrow \pi^{+} \mu^{-} \mu^{-}\right)<4.0 \times 10^{-9} \text { at } 95 \% \text { C.L. [4]. }
$$

This limit is the most restrictive to date [5].
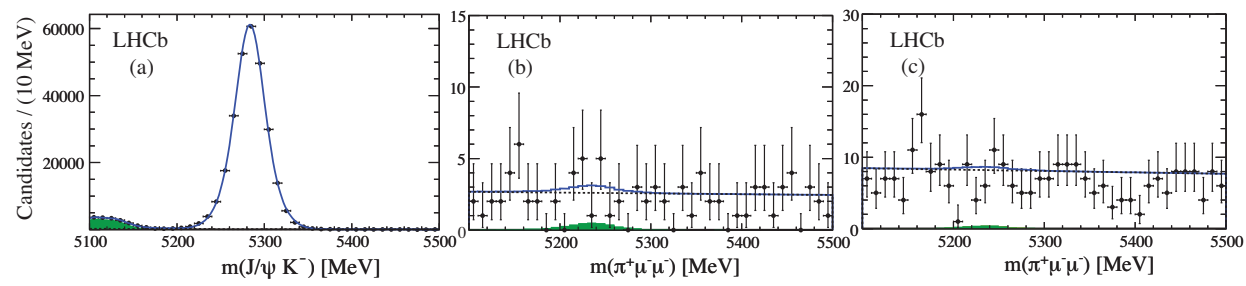

Fig. 3. (Colour on-line) Invariant mass distributions with fits overlaid of candidate mass spectra for $J / \psi K^{-}$(left), $\pi^{+} \mu^{-} \mu^{-}$— for short lifetime (middle), and $\pi^{+} \mu^{-} \mu^{-}$- for long lifetime (right). Backgrounds that peak under the signal in the middle and right-hand side figure are dark grey/green. The dotted lines show the combinatorial backgrounds only, the solid lines show the sum of both backgrounds [4]. 
The two-dimensional plots of the upper limits on $\mathcal{B}\left(B^{-} \rightarrow \pi^{+} \mu^{-} \mu^{-}\right)$for several values of neutrino lifetimes are shown in Fig. 4.

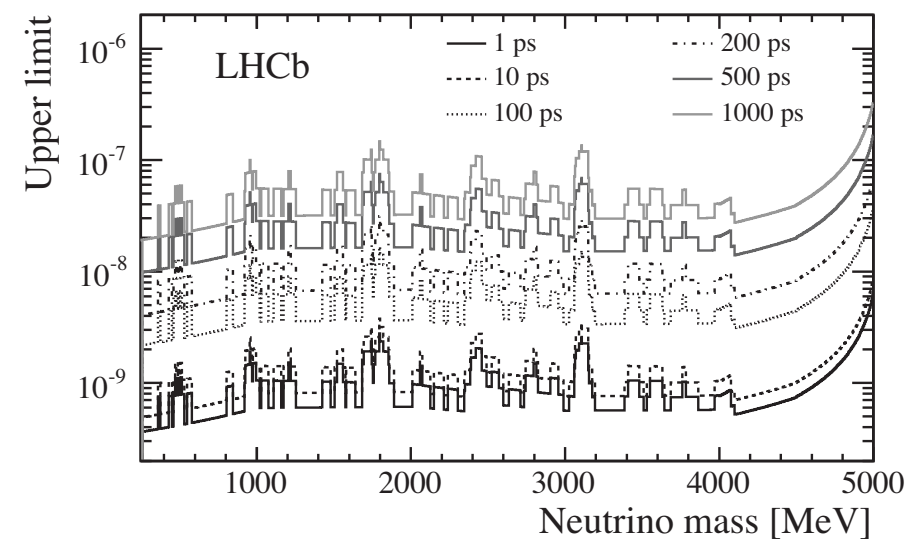

Fig. 4. Upper limits on $B^{-} \rightarrow \pi^{+} \mu^{-} \mu^{-}$decays as a function of neutrino mass and neutrino lifetime $[4,6]$.

\subsection{The search for $B^{-} \rightarrow D^{0} \pi^{+} \mu^{-} \mu^{-}$decay}

The search for the lepton number violating decay $B^{-} \rightarrow D^{0} \pi^{+} \mu^{-} \mu^{-}$ (Fig. 5) was performed with $0.41 \mathrm{fb}^{-1}$ of data collected at a center-of-mass energy of $7 \mathrm{TeV}$. The Majorana neutrino mass range probed is smaller than in the case of $\pi^{+} \mu^{-} \mu^{-}$due to the presence of the $D^{0}$ meson in the final state. The sensitivity of the search is also limited by the need to reconstruct the $D^{0} \rightarrow K \pi$ decay. Peaking backgrounds are essentially absent [5].

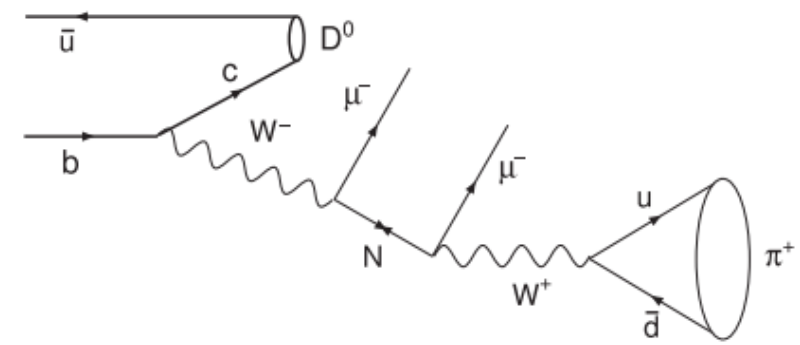

Fig. 5. The Feynman diagram of $B^{-} \rightarrow D^{0} \pi^{+} \mu^{-} \mu^{-}$decay [7].

The invariant mass distribution of $D^{0} \pi^{+} \mu^{-} \mu^{-}$and the upper limit at $95 \%$ C.L. set on Majorana neutrino production as a function of mass are shown in Fig. 6. The largest source of peaking backgrounds is $B^{-} \rightarrow$ $D^{0} \pi^{-} \pi^{-} \pi^{+}$which contributes to only 0.13 events in the signal region. The 
combinatorial background, determined by a linear fit to the sidebands of the $B^{-}$signal region, predicts 35.9 events, while the number of observed is 33 . For Majorana neutrino masses less than $3 \mathrm{GeV} / c^{2}$, the upper limit on the branching fraction is

$$
\mathcal{B}\left(B^{-} \rightarrow D^{0} \pi^{+} \mu^{-} \mu^{-}\right)<1.6 \times 10^{-6} \text { at } 95 \% \text { C.L. [7] }
$$
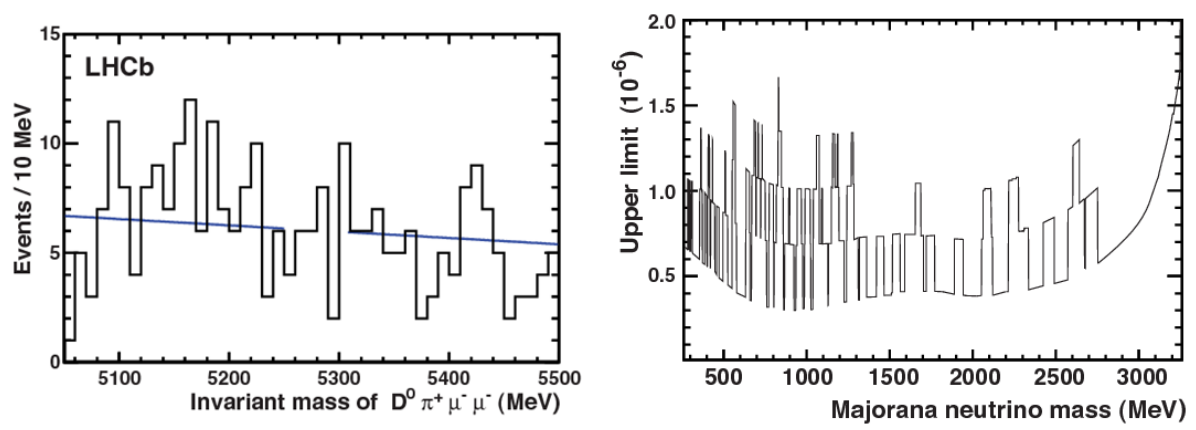

Fig. 6. (Left) Invariant mass distribution of $D^{0} \pi^{+} \mu^{-} \mu^{-}$, (right) upper limit as a function of $\pi^{+} \mu^{-}$mass. The solid line shows the linear fit to the data in the sidebands of $B^{-}$signal region [7].

\subsection{The search for $D_{s}^{+} \rightarrow \pi^{-} \mu^{+} \mu^{+}$decay}

A search for the lepton number violating decay $D_{s}$ meson decay $D_{s}^{+} \rightarrow$ $\pi^{-} \mu^{+} \mu^{+}$(Fig. 7) was performed using $1 \mathrm{fb}^{-1}$ of data collected at a centerof-mass energy of $7 \mathrm{TeV}$.

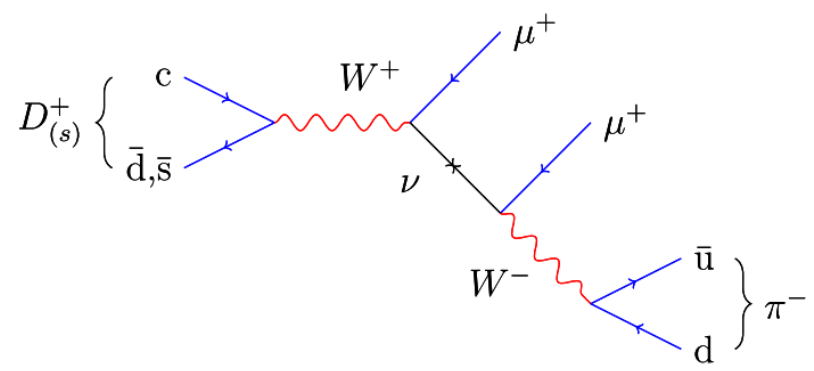

Fig. 7. The Feynman diagram of $D_{s}^{+} \rightarrow \pi^{-} \mu^{+} \mu^{+}$decay.

Candidates selection criteria are applied in order to maximise the expected significance of signal. A boosted decision tree (BDT) distinguishes between signal-like and background-like candidates. The data are split into bins of $m\left(\pi^{-} \mu^{+}\right)$, what improves the statistical significance of any signal 
observed. Figure 8 shows the invariant mass distributions in four mass regions. No signal was found so upper limits on the branching fraction are determined using the $\mathrm{CL}_{s}$ method

$$
\mathcal{B}\left(D_{s}^{+} \rightarrow \pi^{-} \mu^{+} \mu^{+}\right)<1.2 \times 10^{-7} \text { at } 90 \% \text { C.L. [8]. }
$$

The result is the most restrictive to date and represent an improvement by a factor of 50 in comparison to the previous results [6].
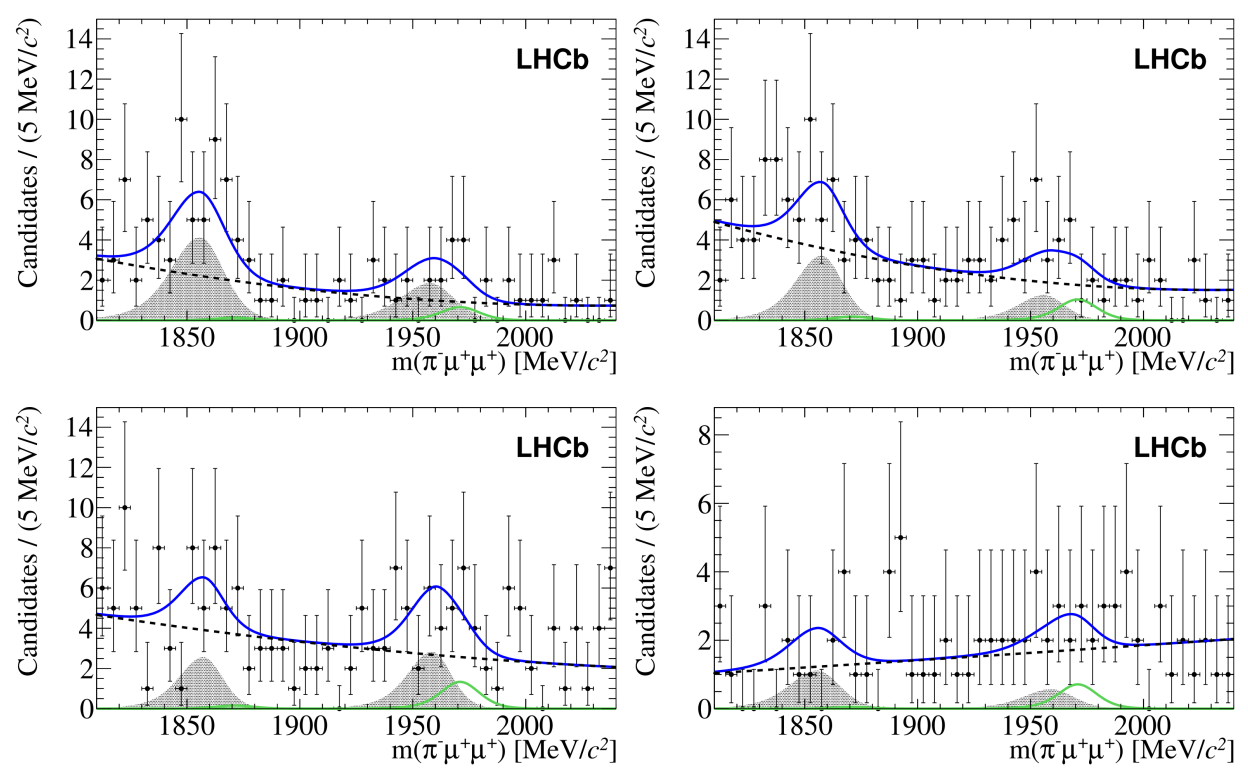

Fig. 8. (Colour on-line) Invariant mass distributions for $D_{s}^{+} \rightarrow \pi^{-} \mu^{+} \mu^{+}+$in the four $m\left(\pi^{-} \mu^{+}\right)$regions. The data are shown as a black point, the signal grey/green line, the peaking background - solid area and the non-peaking background - dashed line [8].

\subsection{The search for $B^{-} \rightarrow \mu^{-} N\left(\rightarrow \pi^{-} e^{+}\right)$decay}

Majorana neutrinos could also be observed by searching for the decay $B^{-} \rightarrow \mu^{-} N\left(\rightarrow \pi^{-} e^{+}\right.$) (Fig. 9), which conserves total lepton number but violates lepton flavour conservation.

With the LHCb Run 1 and Run 2 data sets, a neutrino mass range of $150-4500 \mathrm{MeV} / \mathrm{c}^{2}$ and a lifetime range $1-1000$ ps will be accessible. The normalisation channel is the same as that used in the search for the decay $B^{-} \rightarrow \pi^{+} \mu^{-} \mu^{-}$. In comparison to that analysis, this search will include tracks that start both inside and outside the VELO. 


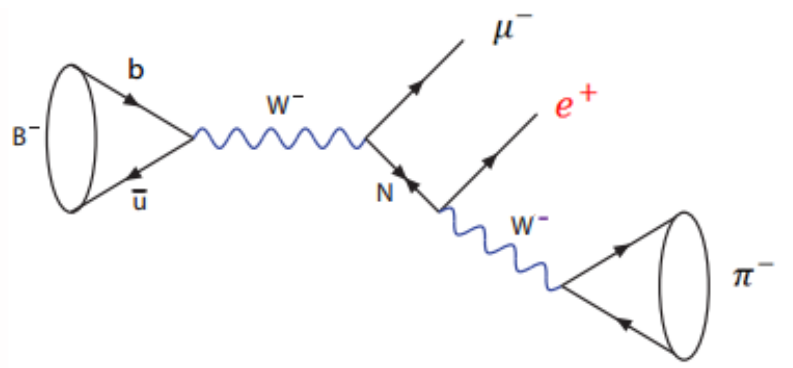

Fig. 9. The Feynman diagram of $B^{-} \rightarrow \mu^{-} N\left(\rightarrow \pi^{-} e^{+}\right)$decay.

\section{Summary}

Searches for Majorana neutrinos using $B$ - and $D$-meson decays with final states that violate total lepton number and/or flavour are potentially sensitive to a large range of neutrino masses and lifetimes. Any observed signal would be an indication of new physics. The searches will be updated and further decay channels will be analyzed with the Run 2 data set.

\section{REFERENCES}

[1] D.H. Perkins, Introduction to High Energy Physics, PWN, Warszawa 2015, ISBN 83-01-14246-4.

[2] LHCb Collaboration, http://lhcb.web.cern.ch/lhcb/

[3] LHCb Collaboration, JINST 3, S08005 (2008).

[4] R. Aaij et al. [LHCb Collaboration], Phys. Rev. Lett. 112, 131802 (2014).

[5] R. Aaij et al. [LHCb Collaboration], Phys. Rev. D 85, 112004 (2012).

[6] X. Cid Vidal, arXiv:1510.05483 [hep-ex].

[7] B. Adeva [LHCb Collaboration], J. Phys.: Conf. Ser. 447, 012062 (2013).

[8] LHCb Collaboration, Phys. Lett. B 724, 203 (2013) [arXiv: $1304.6365 \mathrm{v} 1$ [hep-ex]]. 